\title{
COPD: Wer mehr läuft, lebt besser
}

\author{
Bei COPD-Patienten geht \\ eine Verringerung der \\ körperlichen Aktivität mit \\ einer Verschlechterung \\ der Lebensqualität einher \\ und umgekehrt, d.h. eine \\ Steigerung der körperlichen \\ Aktivität kann zu einer \\ spürbaren Verbesserung der \\ Lebensqualität führen.
}

- 611 konsekutive COPDPatienten einer großen pneumologischen Krankenhausambulanz wurden in die über fünf Jahre laufende Beobachtungsstudie aufgenommen (Durchschnittsalter: 67,2 Jahre, $\mathrm{FEV}_{1}: 49,7 \%$ des Sollwerts, initial negativer Bronchospasmolysetest, keine Exazerbation in den letzten sechs Wochen).

Die Studienteilnehmer füllten u.a. zwei evaluierte krankheitsspezifische Fragebogen zur Lebensqualität aus, den Saint George's Respiratory Questionnaire (SGRQ) und den Chronic Respiratory Questionnaire (CRQ). Zudem wurde mithilfe einer standardisierten Befragung die durchschnittliche Zeit der körperlichen Freizeitaktivität erfasst, insbesondere das Spazierengehen. Aufgrund dieser Befragung wurde die körperliche Aktivität als niedrig ( $<2 \mathrm{~h}$ /Woche), mittel (2-4 h/ Woche) bzw. hoch ( $>4 \mathrm{~h} /$ Woche) klassifiziert.

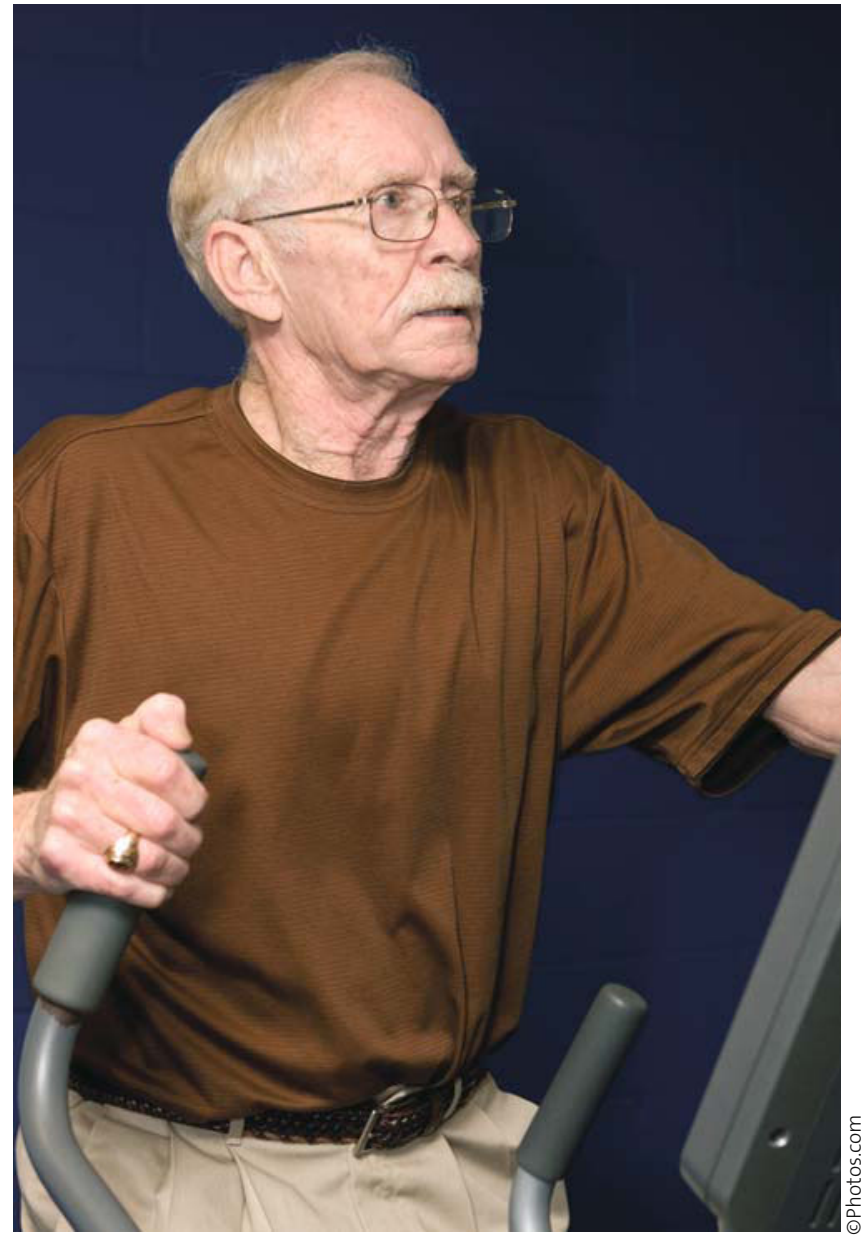

Nach fünf Jahren wurden bei den noch lebenden Patienten $(72,8 \%)$ mit denselben Fragebögen erneut die Lebensqualität und der Grad der körperlichen Aktivität erfasst. Nach Adjustierung bezüglich wichtiger Störfaktoren wie $\mathrm{FEV}_{1}$ oder Komorbiditäten zeigte sich, dass bei den Patienten, die initial eine niedrige körperliche Aktivität aufwiesen, diese aber im Verlauf steigerten, eine deutliche und statistisch hochsignifikante Verbesserung der COPD-spezifischen Lebensqualität zu verzeichnen war.

Bei Patienten hingegen, die eine initial niedrige körperliche Aktivität beibehielten oder die von einem hohen in einen niedrigen Aktivitätsgrad wechselten, fand sich eine signifikante Verschlechterung der Lebensqualitätsscores.

Trainingsfleiß zahlt sich auch bei COPD aus.

\section{Kommentar}

Die Studie sollte uns darin bestärken, unsere COPD-Patienten zu vermehrter körperlicher Aktivität im Alltag zu motivieren, z.B. in Form von regelmäßigen Spaziergängen. Anderseits ist aber festzuhalten, dass lediglich ein kleiner Teil

\section{Promotion / Habilitation. Finden der passenden Professur. www.promotion-d.de} Tel.: 0681 / 7097 689, Fax 0681 / 7097691

West Promotionshilfe $\mathrm{GmbH}$ der Studienteilnehmer auf diese Weise tatsächlich eine Steigerung ihrer körperlichen Aktivität erzielte, (62 der 391 in die Auswertung einbezogenen Patienten = 15,9\%). Daher kann der bloße Rat „Bewegt euch!" nicht als Ersatz für eine leitliniengemäß erforderliche systematische Trainingstherapie gelten. Die Trainingstherapie wird am besten im Rahmen der ambulanten oder stationären Reha begonnen und sollte entweder in Eigenregie oder in einer Lungensportgruppe langfristig fortgesetzt werden.

K. Schultz

\section{- C. Esteban et al.}

Impact of changes in physical activity on health-related quality of life among patients with chronic obstructive pulmonary disease. Eur. Respir. J. 2010 Jan (Epub ahead of print) 